\title{
Characterizing the recovery of a solid surface after tungsten nano- tendril formation
}

G.M. Wright ${ }^{1}$ *, G.G. van Eden ${ }^{2}$, L.A. Kesler ${ }^{1}$, G. De Temmerman ${ }^{2}$, D.G. Whyte ${ }^{1}$, and

\author{
K.B. Woller ${ }^{1}$ \\ ${ }^{1}$ MIT Plasma Science and Fusion Center, 77 Massachusetts Ave. Cambridge, MA, USA, 02139 \\ ${ }^{2}$ FOM Institute DIFFER, Dutch Institute For Fundamental Energy Research, Association \\ EURATOM-FOM, Trilateral Euregion Cluster, Postbus 1207, 3430BE, Nieuwegein, The \\ Netherlands
}

\begin{abstract}
Recovery of a flat tungsten surface from a nano-tendril surface is attempted through three techniques; a mechanical wipe, a $1673 \mathrm{~K}$ annealing, and laser-induced thermal transients. Results were determined through SEM imaging and elastic recoil detection to assess the helium content in the surface. The mechanical wipe leaves a $\sim 0.5 \mu \mathrm{m}$ deep layer of nanotendrils on the surface post-wipe regardless of the initial nano-tendril layer depth. Laserinduced thermal transients only significantly impact the surface morphology at heat loads of $35.2 \mathrm{MJ} / \mathrm{m}^{2} \mathrm{~s}^{1 / 2}$ or above, however a fully flat or recovered surface was not achieved for 100 transients at this heat load despite reducing the helium content by a factor of $\sim 7$. A $1673 \mathrm{~K}$ annealing removes all detectable levels of helium but sub-surface voids/bubbles remain intact. The surface is recovered to a nearly flat state with only some remnants of nano-tendrils re-integrating into the surface remaining.
\end{abstract}

PACS: 52.40.Hf, 81.07-b, 81.05.Je,

PSI-21 keywords: Helium, Ion-surface interactions, Thermal shock, W fuzz 
*Corresponding author address: 77 Massachusetts Ave. NW17-121, Cambridge, MA, USA, 02139.

*Corresponding author email: wright@psfc.mit.edu

Presenting author: G.M. Wright

Presenting author email: wright@psfc.mit.edu 


\section{Introduction}

The growth of tungsten (W) nano-tendrils, or "fuzz", on surfaces at high temperatures $(>1000 \mathrm{~K})$ exposed to helium $(\mathrm{He})$ plasma ions is well established in linear devices $[1,2$, 3] as well as tokamaks [4]. The exact growth mechanism and impact of these tendrils is still under debate. In an experimental device, such as ITER, if plasma conditions where nano-tendril growth can occur are accessed in the $\mathrm{W}$ divertor, removal techniques, preferably in-situ, will be desired to recover a pristine W surface.

Studies have shown several methods to "recover" from a nano-tendril surface (i.e. removal of nano-tendrils from a tungsten surface). Due to the mechanical fragility of W nano-tendrils, mechanical removal of the tendrils is an option [5] and will leave the bulk material unaffected. Nano-tendrils have also been found to re-integrate into the surface from high temperature annealing [5-8], which releases the He trapped in the W. Finally, thermal transients on the surface have also been shown to modify nano-tendril structures $[3,9]$.

These different surface "recovery" techniques have subtle differences. The mechanical removal simply removes the tendrils but has no effect on the bulk. The high temperature anneal heats the tendrils and the bulk equally. The thermal transients deposit most of their energy, and thus induce temperature increases mostly in the tendrils themselves while the temperature of the majority of the $\mathrm{W}$ bulk is unaffected. The purpose of this study is to compare the effects of these three surface recovery techniques in terms of the changes to the surface and sub-surface morphologies as well as the $\mathrm{He}$ content of the targets. Differences observed between these techniques will give insight into the mechanisms leading to tendril removal or re-integration into the surface. This, in 
turn, may provide further clues as to the mechanisms that induce the nano-tendril growth initially.

\section{Experiment}

a. Mechanical removal of $\mathrm{W}$ nano-tendrils

The nano-tendril surfaces to be recovered via mechanical wipe were grown in the helicon plasma source of DIONISOS [1이. The nano-tendril surfaces were grown on a polished $99.95 \mathrm{at} \%$ pure $\mathrm{W}$ target. The targets were exposed to pure He plasma for $3600 \mathrm{~s}$ and plasma flux of $(3 \pm 1) \times 10^{22} \mathrm{~m}^{-2} \mathrm{~s}^{-1}$. One target was exposed at a temperature of $1250( \pm$ 100) $\mathrm{K}$ and a bias of $-60 \mathrm{~V}$ and a second target was exposed at a target temperature of $1400( \pm 50) \mathrm{K}$ and a bias of $-90 \mathrm{~V}$.

The mechanical wipe was performed on a Buehler Ecomet 3000 polisher with an Automet 2 head. A Chemia MB fine polishing cloth was mounted on the Ecomet 3000 rotating at $10 \mathrm{rpm}$. The $\mathrm{W}$ nano-tendril sample was mounted on the Automet 2 head at 60 rpm in co-rotation with the base. The $\mathrm{W}$ nano-tendril surface was pressed into the polishing cloth with $8.9 \mathrm{~N}$ of perpendicular force for 3 seconds.

The surface and sub-surface morphology were examined with SEM and focused ion beam cross-sectioning. Images were taken before and after the mechanical removal of the nano-tendrils. The He content of the surface was measured via heavy ion elastic recoil detection (ERD) [11]. For this work, a $6 \mathrm{MeV} \mathrm{O}^{3+}$ ion beam was used with a scattering angle of $35^{\circ}$. A $6 \mu \mathrm{m}$ mylar foil was placed in front of the solid-state detector to block the reflected $\mathrm{O}^{3+}$ but allow the forward-scattered $\mathrm{He}$ and hydrogen from the surface to pass through. With this set-up and these $\mathrm{O}^{3+}$ energies, the He concentration can be measured across an areal density of $\sim 3 \times 10^{17} \mathrm{~W} / \mathrm{cm}^{2}$, which is equivalent to a depth of $50 \mathrm{~nm}$ in 
bulk $\mathrm{W}$ or $500 \mathrm{~nm}$ of $\mathrm{W}$ fuzz if it is assumed $\sim 90 \%$ porosity for $\mathrm{W}$ nano-tendril layers. The He content in this layer is quantified by using simNRA [12] to fit experimental spectra. The He content detection limit for the ERD conditions used in this work is $\sim 0.1$ at.\%. Previous work [11] has found that the He concentration is roughly constant within the nano-tendril layer and that holds true for this work as well (i.e. He concentration for the entire detection depth falls within the error bars of Fig. 4).

b. High temperature annealing of $\mathrm{W}$ nano-tendril surfaces

The $\mathrm{W}$ nano-tendril surfaces for the high temperature annealing were also grown in DIONISOS under similar plasma fluxes and conditions for $3600 \mathrm{~s}$. One target was exposed at a temperature of $1200( \pm 50) \mathrm{K}$ and a bias of $-70 \mathrm{~V}$ and a second target was exposed at a target temperature of $1450( \pm 50) \mathrm{K}$ and a bias of $-80 \mathrm{~V}$.

Both targets were annealed at the same time. The annealing was performed in a R.D. Webb vacuum furnace. The furnace temperature was ramped at a rate of $1 \mathrm{~K} / \mathrm{s}$ to $1673 \mathrm{~K}$ and held at that temperature for $3600 \mathrm{~s}$.

The same procedures to investigate surface and sub-surface morphologies and surface $\mathrm{He}$ content from section 2a were used on the annealed targets.

c. Transient heating of $\mathrm{W}$ nano-tendril surfaces via laser pulse

The $\mathrm{W}$ nano-tendril surface for transient heating was grown in PILOT-PSI [3]. The $\mathrm{W}$ target was exposed to pure He plasma with a central flux density of $9 \times 10^{23} \mathrm{~m}^{-2} \mathrm{~s}^{-1}$ for a total of $630 \mathrm{~s}$ distributed over 9 equal plasma discharges. The surface temperature during plasma exposure was $1500 \pm 50 \mathrm{~K}$ and the target was biased at $-38 \mathrm{~V}$.

Different spots of the nano-tendril surface were exposed to different thermal transients. In this study we investigate three spots denoted by A, B, and C. The conditions 
for each laser spot are defined by power, heat flux factor $\left(\mathrm{F}_{\mathrm{HF}}\right)$ and the number of pulses (see Table 1). All laser pulses are $1 \mathrm{~ms}$ in duration, which results in a heat penetration skin depth of $\sim 50 \mathrm{~nm}$ in bulk $\mathrm{W}$ and $\sim 500 \mathrm{~nm}$ in a $90 \%$ porous nano-tendril layer. The temperature rise $(\Delta \mathrm{T})$ of the surface was measured by high-speed IR camera $(\mathrm{SC} 7500 \mathrm{M}$, FLIR Systems Inc, 1.5-5.1 $\mu \mathrm{m}$ ) and the bulk tungsten was kept at room temperature. On the initial laser pulse, where an unmodified, fully formed nano-tendril layer with emissivity of $0.8[13]$ is assumed, $\Delta \mathrm{T}=3100 \pm 200 \mathrm{~K}$ for all three spots. In the absence of a method to measure the surface emissivity directly we have used estimates from previous work, however any over- or under-estimation of the emissivity will have an impact on the measured $\Delta \mathrm{T}$. The temperature rise for subsequent pulses and the average for the total number of pulses are lower than for the initial pulse but difficult to quantify accurately as the absorbance and emissivity of the surfaces can change as the surface morphology evolves. More details on the temperature measurement techniques can be found in [14] and [15].

Analysis techniques of the surface are similar to sections $2 a$ and $2 b$, but with some key differences. Focused ion beam cross-sectioning and SEM imaging was used on the laser spots to investigate the final state of the surface and the nano-tendrils just outside the laser spot (but at a similar radial location on the target) are assumed to be representative of the surface before the laser heating. This was also assumed for the $\mathrm{He}$ content ERD measurements. A spot just outside the laser spot is assumed to be the unperturbed He content of the nano-tendrils on the surface. To measure the He content of the surface after the laser pulses, a $0.3 \mathrm{~mm}$ beam aperture was used such that the entire beam spot could fit within the $\sim 2 \mathrm{~mm}$ diameter laser spot. 


\section{Results and Discussion:}

\section{a. Mechanical removal of $\mathrm{W}$ nano-tendrils}

The SEM images show that the mechanical wipe was unsuccessful in fully removing the nano-tendril structures from the surface for both $1250 \mathrm{~K}$ and $1400 \mathrm{~K}$ exposure temperatures (see Fig. 1). From the cross-section images it is seen that the initial fuzz layer was significantly thicker for the $1400 \mathrm{~K}$ exposure $(\sim 2 \mu \mathrm{m}$ layer depth) than for the $1250 \mathrm{~K}$ exposure ( $\sim 0.8 \mu \mathrm{m}$ layer depth), but the remaining fuzz layer after the mechanical wipe was approximately the same for both samples $(\sim 0.4 \mu \mathrm{m})$. This indicates that, for these growth conditions, there is a defined "base layer" (i.e. the first $0.4 \mu \mathrm{m}$ of the nanotendril layer) of the tendril structures that has significantly higher mechanical strength than the tendril structures that grow beyond this base layer regardless of the overall nanotendril layer depth. It appears that the nano-tendril layer is woven more tightly or has a lower overall porosity closer to the bulk substrate, which may result in its increased mechanical strength. This is consistent with other findings that show that overall nanotendril layer porosity increases as the layer grow thicker [1ㅁ] and as the tendrils extend further from the W bulk [17]. The mechanical removal of nano-tendrils in [5] was more effective and removing the entire nano-tendril layer, however, the nano-tendril layer in [5] was grown at lower $\mathrm{W}$ temperatures and $\mathrm{He}$ ion energies. This resulted in smaller diameter tendrils meaning they are more fragile and prone to mechanical removal.

Measurements with ERD before and after the mechanical wipe show that the $\mathrm{He}$ content of the surface is relatively unchanged (see Fig. 4), as one would expect since nano-tendril structures remain on the surface. The nano-tendril layer grown at $1400 \mathrm{~K}$ actually shows a small increase in He content after the mechanical wipe. This is 
consistent with the He profile from before the wipe as it had a slightly increasing $\mathrm{He}$ content deeper into the nano-tendril layer. The increasing He content is consistent with a lower porosity nano-tendril base with larger tendril diameters since it will make it more unlikely for He bubbles/voids to grow and burst through a surface.

b. High temperature annealing of $\mathrm{W}$ nano-tendrils

The SEM images show that the $1673 \mathrm{~K}$ annealing almost completely removed all nanotendril morphology from the surface (see Fig. 2). It is noteworthy that $1673 \mathrm{~K}$ is in the recrystallization range for tungsten and this may also play a role in the re-integration of the nano-tendrils back into the surface. After the annealing, there are still some large diameter individual tendrils remaining on the surface. These remaining tendrils are typically $200-400 \mathrm{~nm}$ in diameter, meaning they are a factor of 10-20 times larger diameter than the nano-tendrils on the surface before the annealing. It is likely these are remnants of collapsed nano-tendril structures that conglomerate together before receding back into the surface. Similar behaviour was observed in [6] and it is probable that a longer annealing or a higher annealing temperature would remove these residual structures and recover a fully flat surface.

The ERD measurements show that annealing at $1673 \mathrm{~K}$ has removed the He from the surface up to the detection limits of the technique $(\sim 50 \mathrm{~nm}$ of bulk W). However, cross-section images show that there are still voids/bubbles present in the subsurface region, some of which are within the first $50 \mathrm{~nm}$ of $\mathrm{W}$ and thus within the ERD detection depth. This indicates that while this anneal temperature is high enough to thermally release $\mathrm{He}$ and reduce $\mathrm{He}$ content to below our detection limit ( 0.1 at.\%), it is not high enough to anneal out the voids themselves. This may imply that it is the presence of $\mathrm{He}$ in 
combination with the presence of voids, rather than the voids themselves that stabilize the nano-tendrils. Due to the limitations of our detection depth we cannot confirm that $\mathrm{He}$ content of the He bubbles deeper than $50 \mathrm{~nm}$ into the bulk also have reduced He content but since the annealing is a bulk heating process, it seems likely that all $\mathrm{He}$ bubbles would be affected similarly regardless of depth in the surface.

c. Transient Heating of W nano-tendrils via laser heating

The SEM images show that only spot A, with $\mathrm{F}_{\mathrm{HF}}=35.2 \mathrm{MJ} / \mathrm{m}^{2} \mathrm{~s}^{1 / 2}$, was effective at conglomerating and re-integrating some of the nano-tendril structures from the surface (see Fig. 3), but even at these heat loads, the surface morphology is not removed to the extent that was seen from the $1673 \mathrm{~K}$ anneal (see Sec. 3b). The heat penetration skin depth may not reaching all the way to the base of the nano-tendril layer, but this would depend on the exact porosity of the nano-tendril layer. If the nano-tendril layer is $90 \%$ porous, for example, then heat penetration is only $\sim 500 \mathrm{~nm}$ and may not reach the base of the nano-tendril layer, but if porosity is closer to $95 \%$ then heat penetration is $\sim 1000 \mathrm{~nm}$ which should penetrate the entire nano-tendril layer. However, since porosity is not measured directly, no definitive conclusions can be made about how far the heat penetrates into the nano-tendril layer. All the thermal transient spots did have an effect on the bulk $\mathrm{W}$ for all three spots, which would indicate that the heat penetration is reaching the bulk for at least some of the transients and implies a porosity of $>90 \%$. SEM imaging shows significant surface roughening for all spots investigated and development of a crack network for spot C, which was exposed to 10000 thermal transient events. Similar roughening been seen for similar thermal transients on bulk W, however the crack network only develops on targets pre-exposed to He plasma [15]. For the lower power 
thermal transients (spots B and C), the nano-tendril diameter increased slightly after the thermal transients but the overall nano-tendril layer depth was unchanged for B (1000 transients) and only minor modifications for C (10 000 transients). However, it has been observed for nano-tendrils grown at lower temperatures, the tendril layer depth can be reduced by a factor of $\sim 2$ for $\mathrm{F}_{\mathrm{HF}}=19 \mathrm{MJ} / \mathrm{m}^{2} \mathrm{~s}^{1 / 2}[15]$.

The ERD detection depth is similar to the skin depth of the laser pulse heating, both being $\sim 50 \mathrm{~nm}$ in bulk W. So, we can be confident that we are measuring the full impact of the laser pulses on the He concentration in the surface despite the shallow detection depth of the ERD measurement. The ERD spectra show that He remains in the tungsten as we would expect since tendril structures still exist within all the spots investigated. However, the transients did have an impact. For the A, where the transient heat load is greatest and the impact on the nano-tendril morphology is most pronounced, the He content in the surface is reduced by a factor of $\sim 7$. For B and C, the He in the surface is reduced by a factor of $\sim 3$ and $\sim 4$ respectively (see Fig. 4). Spot $C$ had a factor of 10 more thermal transients than B indicating a diminishing impact on He content with additional thermal transients. However, spot A showed significant changes after only 100 transients, so additional transients may continue to reduce the He content and prompt a more complete recovery of the surface and re-integration of the nano-tendrils.

\section{Conclusions}

Of the three recovery techniques investigates, the $1673 \mathrm{~K}$ anneal was the most effective at recovering a flat surface. The annealing resulted in a nearly fully recovered surface with only occasional individual thickened tendrils remaining on the surface. These large diameter tendrils are thought to be remnants from conglomerated nano-tendril structures 
before they are re-integrated into the surface. ERD analysis did show that the implanted He was fully released (or below the detection limit of the technique). However this technique is the most difficult and impractical to incorporate as an in-situ method into a future fusion device.

The thermal transients induced by laser pulse only impacted the nano-tendril layer for heat loads of $35.2 \mathrm{MJ} / \mathrm{m}^{2} \mathrm{~s}^{1 / 2}$ or higher, which is in the range where $\mathrm{W}$ melting becomes a concern. At these transient heat loads, the surface morphology is not completely removed but the fine nano-tendrils do seem to have conglomerated into a coarser morphology. After 100 transients, the He content in the surface was reduced by a factor of $\sim 7$ but not to zero. It is thought that additional transients could further reduce the He content in the surface to zero and this could results in a full surface recovery similar to the $1673 \mathrm{~K}$ anneal. An added benefit of this technique is that it is the most easily adapted into an in-situ removal technique using radiating plasma terminations, ELMs, or flash lamps on a robotic arm.

Finally, the mechanical wipe proved to be the least effective method of removing nano-tendrils from the surface. After a defined mechanical wipe, a nano-tendril layer $\sim 0.5$ $\mu \mathrm{m}$ deep remained regardless of the initial nano-tendril layer depth. This indicates the "base layer" of the nano-tendrils, which appears to be less porous than the rest of the nano-tendril layer, is more mechanically robust than the nano-tendril tips and is not easily removed by the simple mechanical wipe employed in this study. It is possible that applying more force to the wipe or more thorough and persistent wiping would eventually remove even this base layer of nano-tendrils. A mechanical wipe could be performed in-situ with a robotic arm, however the wipe parameters (e.g. applied force, 
wiping surface, etc) would need to be optimized to remove all nano-tendril layers completely while removing as little $\mathrm{W}$ bulk as possible to limit the impact on component lifetime.

\section{Acknowledgements}

This work is supported by US DOE award DE-SC00-02060. This work made use of the MRSEC Shared Experimental Facilities at MIT, supported by the National Science Foundation under award number DMR-08-19762.

\section{References}

[1] M.J. Baldwin, R.P. Doerner, Nucl. Fusion 48 (2008) 035001.

[2] S. Kajita, S. Takamura, N. Ohno, D. Nishijima, H. Iwakiri, N. Yoshida, Nucl. Fusion 47 (2007) 1358.

[3] G. De Temmerman, K. Bystrov, R.P. Doerner, L. Marot, G.M. Wright, K.B. Woller, D.G. Whyte, J.J. Zielinski, J. Nucl. Mater. 438 (2013) S78.

[4] G.M. Wright, D. Brunner, M.J. Baldwin, R.P. Doerner, B. Labombard, B. Lipschultz, J.L. Terry, D.G. Whyte, Nucl. Fusion 52 (2012) 042003.

[5] M.J. Baldwin, R.P. Doerner, J. Nucl. Mater. 404 (2010) 165.

[6] M. Yajima, N. Yoshida, S. Kajita, M. Tokitani, T. Baba, N. Ohno, J. Nucl. Mater. 449 (2014) 9.

[7] S. Kajita, N. Yoshida, R. Yoshihara, N. Ohno, T. Yokochi, M. Tokitani, S. Takamura, J. Nucl. Mater. 421 (2012) 27.

\section{[8] New ref}

[9] S. Kajita, N. Ohno, W. Sakaguchi, M. Takagi, Plasma Fus. Res. 4 (2009) 004. 
[10] G.M. Wright, H.A. Barnard, L.A. Kesler, E.E. Peterson, P.W. Stahle, R.M. Sullivan, D.G. Whyte, K.B. Woller, Rev. Sci. Instr. 85 (2014) 023503.

[11] K.B. Woller, D.G. Whyte, G.M. Wright, R.P. Doerner, G. De Temmerman, J. Nucl. Mater. 438 (2013) S913.

[12] M. Mayer, Technical Report IPP 9/113, Max-Planck-Institut für Plasmaphysik, Garching, Germany (1997).

[13] S. Kajita, G. De Temmerman, T.W. Morgan, G.G. van Eden, T. de Kruif, N. Ohno, Nucl. Fusion 54 (2014) 033005.

[14] G.G. van Eden, T.W. Morgan, H.J. van der Meiden, J. Matejicek, T. Chraska, M. Wirtz, G. De Temmerman, Nucl. Fusion 54 (2014) 123010.

[15] G. De Temmerman, T.W. Morgan, G.G. van Eden, T. de Kruif, M. Wirtz, J. Matejicek, T. Chraska, R.A. Pitts, G.M. Wright, "Effect of high-flux H/He plasma exposure on tungsten damage due to transient heat loads", in these proceedings

[1ㅌ] D. Nishijima, M.J. Baldwin, R.P. Doerner, J.H. Yu, J. Nucl. Mater. 415 (2011) S96.

[1]] S. Kajita et al, J. Nucl. Mater. 421 (2012) 22. 
Table captions:

Table 1: Details of thermal transient conditions for laser spots.

Tables:

Table 1:

\begin{tabular}{||c|c|c|c|c|}
\hline Spot & $\begin{array}{c}\text { Power } \\
\left(\mathrm{GW} / \mathrm{m}^{2}\right)\end{array}$ & $\begin{array}{c}\mathrm{F}_{\mathrm{HF}} \\
\left(\mathrm{MJ} / \mathrm{m}^{2} \mathrm{~s}^{1 / 2}\right)\end{array}$ & $\begin{array}{c}\underline{\# \text { of }} \\
\text { pulses }\end{array}$ & $\underline{\underline{\text { Pulse }}}$ \\
A & 1.11 & 35.2 & $\underline{100}$ & $\underline{10}$ \\
\hline B & 0.61 & 19.4 & $\underline{1000}$ & $\underline{40}$ \\
\hline C & 0.62 & 19.5 & $\underline{10000}$ & $\underline{40}$ \\
\hline
\end{tabular}




\section{Figure Captions:}

Figure 1: Normal and cross-section SEM images before and after a mechanical wipe of $\mathrm{W}$ nano-tendril surfaces grown at a) $\mathrm{T}_{\text {surf }}=1250 \mathrm{~K}$, and b) $1400 \mathrm{~K}$. Viewing angle is $52^{\circ}$ for cross section images.

Figure 2: Normal and cross-section SEM images before and after a $1673 \mathrm{~K}$ anneal of W nano-tendril surfaces grown at a) $\mathrm{T}_{\text {surf }}=1250 \mathrm{~K}$, and b) $1400 \mathrm{~K}$. Viewing angle is $52^{\circ}$ for $\underline{\text { cross section images. }}$

Figure 3: Normal and cross-section SEM images before and after thermal transients to W nano-tendril surfaces laser spots a) A, b) B, and c) C (see Table 1). The dashed lines approximate the transition from nano-tendril layer to bulk. Viewing angle is $52^{\circ}$ for cross $\underline{\text { section images. }}$

Figure 4: He concentration as measured by ERD in W nano-tendril surfaces before and after undergoing various surface recovery techniques. The first $3 \times 10^{17} \mathrm{~W} / \mathrm{cm}^{2}$ of the surface corresponds to $\sim 50 \mathrm{~nm}$ in bulk W or $\sim 500 \mathrm{~nm}$ in a $90 \%$ porous nano-tendril layer. 
Figures

P3-010

Figure 1:
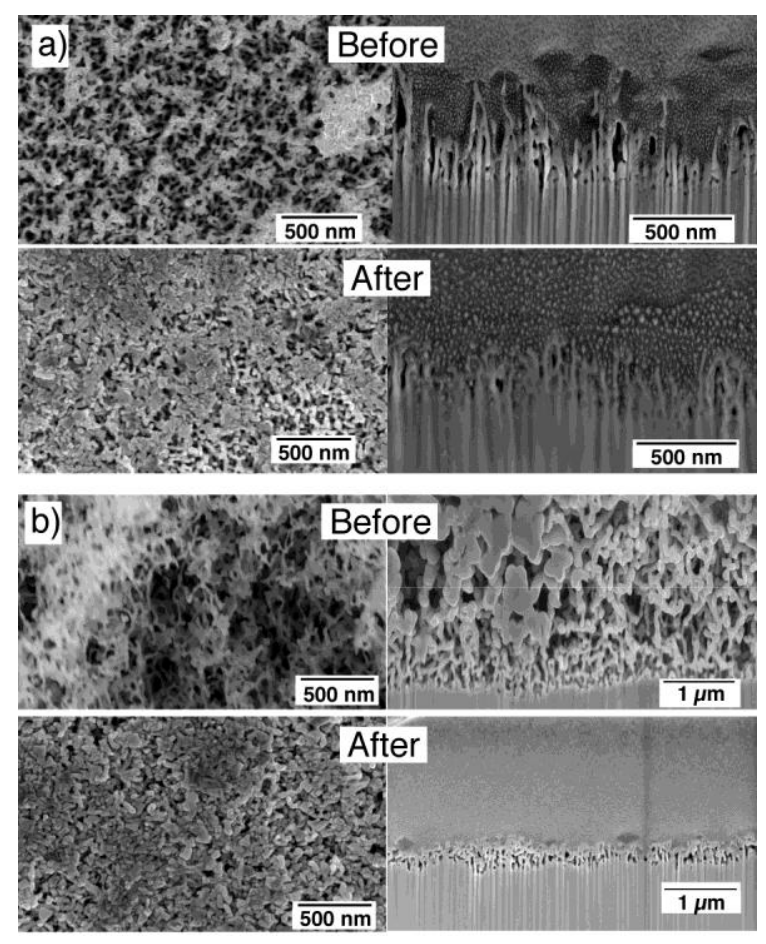
Figure 2:

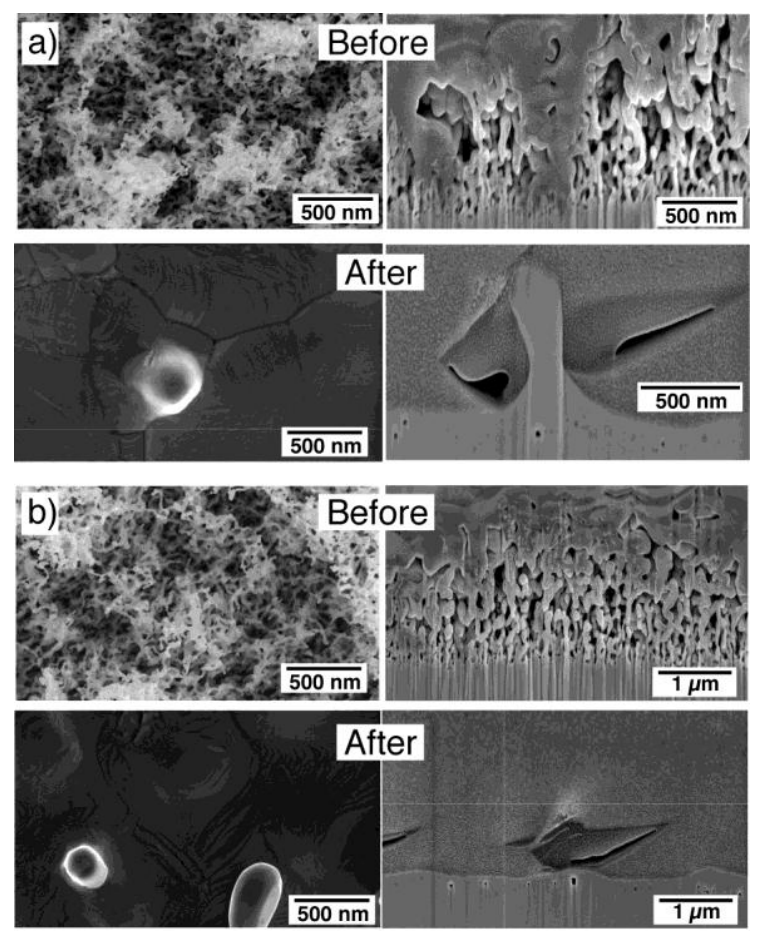


Figure 3:
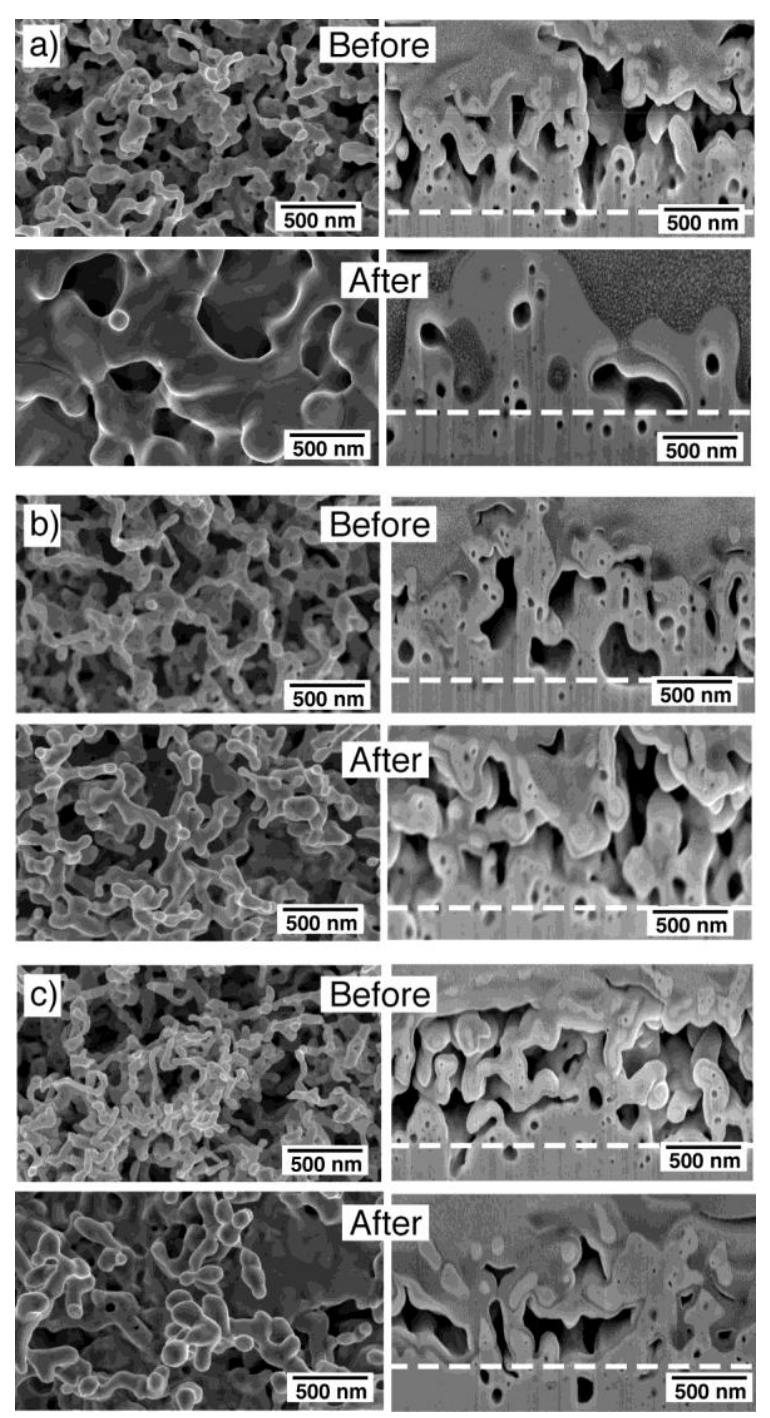
Figure 4:

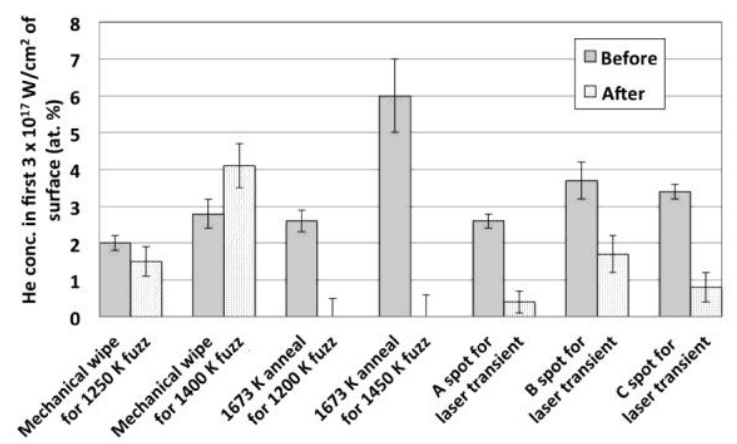

